\title{
Effect of Impact Angle on Particle Fracture Phenomenon
}

Aerosol and Air Quality Research

\author{
Hiroshi Satone*, Kenji limura, Michitaka Suzuki \\ Department of Chemical Engineering, Graduate School of Engineering, University of Hyogo, \\ Hyogo 671-2280, Japan
}

\section{ABSTRACT}

The effect of terephthalic acid particle properties on particle fracture phenomena was investigated in this study. Furthermore, to evaluate the fracture characteristics in real process, the effect of the particle impact angle on particle fracture phenomena was also investigated. The results of this study indicated that: (i) the crystallite size correlated with the fracture stress of the particle; (ii) the crystallite size also showed a correlation with the critical fracture velocity and the kinetic energy of the particle; and (iii) the particle fractured more easily at impact angles under $45^{\circ}$.

Keywords: Particle strength, Particle fracture, Impact angle, Pneumatic transportation, Terephthalic acid

\section{INTRODUCTION}

In various industrial processes involving particle movement, such as a powder pneumatic transportation system and a solid-gas septarian using air filtration, particles often fracture because of the impact with a pipe's inner wall or with the surface of a fiber in an air filter. Particle fracturing may cause several problems, such as an increase in the apparent volume, clogging of the powder transfer pipe, and deterioration in flowability because of a relative increase in the adhesion force. Additionally, this phenomenon may significantly affect a subsequent process and the final product quality. Thus, the investigation of particle fracturing is very important.

Research on the particle fracture phenomenon, especially single-particle fracturing, has been conducted for a long time. For example, Rittinger's or Kick's research on pulverization energy includes an extremely wide range of studies that are related to pulverization energy measurements or compression fracture loads (Pitchumani et al., 2004; Kanda, 1996), as well as an investigation of the effect of particle diameter and structure on the compression strength (Griffith, 1924; Yashima and Saito, 1979; Golchert et al., 2004). Moreover, the kinetic point of view, including the investigation of fracturing behavior of particles under an impact load without considering the stationary fracture phenomenon, has been presented in some studies (Chaudhri, 2004; Chau et al., 2000; Khanal et al., 2005).

In consideration of the above, although single-particle fracturing has been studied in detail, the design of real industrial process applications is based on past experiences. One of the main reasons is the fact that most of the previously conducted research studies have only considered the limited case of uniaxial compression. In most cases, previous research studies cannot be directly applied to real processes. The granule fracturing mechanism under biaxial compression conditions was analyzed in our previous report (Satone et al., 2017) using both experimental and simulation methods. The results of this study indicated that (1) the fracturing behaviour differs, depending on the number of compression axes, and (2) the fracture load under the biaxial condition is smaller than that under the uniaxial condition. Nevertheless, even applying these results to real processes, some issues remain since our previous report was conducted under ideal conditions.

The particle fracture phenomenon has been investigated in several studies using simulations. The discrete element method reported in (Samimi et al., 2005; Bolton et al., 2008) considers a 
particle as a collection of fine particles. In other simulations, the finite element method was applied for individual particle fracturing by calculating the stress distribution within the particles under compression (Tsoungui et al., 1999; Gundepudi et al., 1997). Although simulations are useful in modeling particle fracturing, they also exhibit several issues because of the restrictive conditions imposed during modeling. Thus, several current useful studies cannot be directly applied to practical problems.

Alternatively, the dynamic particle fracture phenomenon, which is more complex than the normal fracture phenomenon, has been analyzed under static or quasi-static conditions. Processes under real conditions could not be analyzed because of their difficulty of observation. In many research studies, a particle was forced to impact a wall vertically, even if the experimental conditions were dynamic. The effect of the impact angle has received less attention. In our previous research work on single-particle fracturing under biaxial conditions (Satone et al., 2017), the experimental results indicated that the fracture load under biaxial conditions differs, depending on the direction of the resultant force vector in the two axes. This result is very important in investigating the effect of impact angle on the particle fracture phenomenon. Moreover, the particle fracturing characteristics can be predicted from any physical property value of the particle without using empirical laws.

This paper aims to analyze the particle fracture phenomenon under conditions close to those encountered in real processes. Particle impact tests are conducted for different impact velocities and impact angles using a modified cascade impactor. Furthermore, the relation between the particle fracture phenomenon and particle properties is investigated by conducting uniaxial and biaxial single-granule compression tests and the X-ray powder diffraction method.

\section{EXPERIMENTAL METHODS}

\subsection{Sample Powder}

A terephthalic acid powder was used as a test sample. A large quantity of this powder was used as a raw material for the polyethylene terephthalate (PET) resin. Reportedly, problems are caused by particle fracturing in a pneumatic transportation process at a PET manufacturing plant. Four samples of terephthalic acid powder with a $1520 \mathrm{~kg} \mathrm{~m}^{-3}$ density obtained from four different manufacturers (Sample A: Kishida Chemical Co., Ltd.; Sample B: Tokyo Chemical Industry Co., Ltd.; Sample C: Merck KGaA; and Sample D: Kanto Chemical Co., Inc.) were used in the experiments. Fig. 1 shows the particle size distribution of each powder sample.

\subsection{Evaluation of Physical Properties}

The physical properties of particles, such as particle size distribution, crystallite size, and

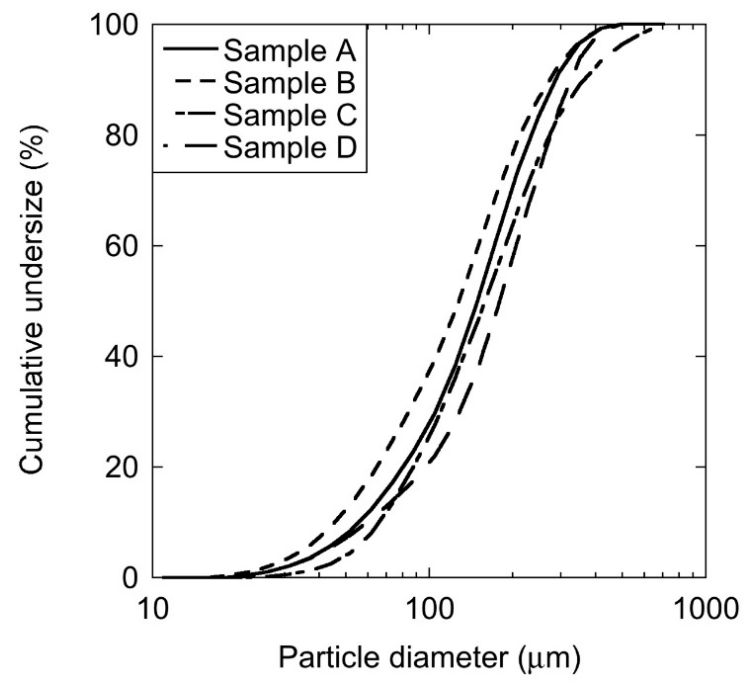

Fig. 1. Particle size distributions for various terephthalic acid sample. 
fracture stress, were measured. The particle size distribution was measured using a laser diffraction method (MicrotracBEL Corp., Microtrac FRA). The crystallite size was calculated using a powder $\mathrm{X}$-ray diffraction (XRD) pattern. This pattern was determined via powder XRD using a diffractometer (Rigaku Corp., MiniFlex II) equipped with a $\mathrm{Cu}-\mathrm{K} \alpha$ radiation source. The scan rate was $2.0^{\circ} \mathrm{min}^{-1}$, the step interval was $0.02^{\circ}$, and the scan range was in the $10^{\circ}-70^{\circ} 2 \theta$ range. The fracture stress measurement of a single particle was based on the JIS R1639-5 (JIS R1639-5, 2007) using a microcompression tester (Shimazu Crop., MCT-510). The particles were compressed to the point of fracture, and the fracture load $F_{1}(\mathrm{~N})$ was recorded. One hundred particles were used in each compression test. The fracture stress of a single particle $S(\mathrm{~Pa})$ was calculated using the following Hiramatsu's equation (Hiramatsu et al., 1965) for a fracture load and a particle diameter $x(\mathrm{~m})$ :

$S=\frac{2.8 F_{1}}{\pi x^{2}}$

\subsection{Particle Impact Test}

Fig. 2 shows a schematic diagram of the particle impact testing apparatus. The system consists of a single stage of the cascade impactor (C1-150, Shimazu Corp.), a membrane filter, and a gas suction pump. An angle-adjustable plate is used at the impact section of the cascade impactor. Using this mechanism, a particle can be impacted on the plate for a wide range of impact velocities and impact angles. The distance from the nozzle outlet of the cascade impactor to the impact plate is $5.5 \mathrm{~mm}$. The particle impact test process is described as follows. Initially, the gas suction pump was set to a constant flow rate. The particles were poured into the inlet of the cascade impactor. After the pouring was completed, almost all particles in the cascade impactor and membrane filter were collected. A change of $50 \%$ particle diameter was evaluated. Table 1 summarizes the nozzle size, the flow velocity at the nozzle, and the theoretical cut off diameter of $50 \%$ collection. Theoretically, the expansion of gas passing through the nozzle must be

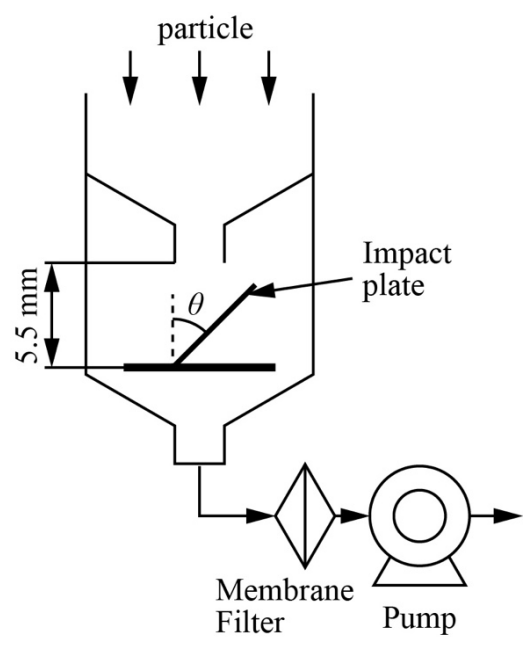

Fig. 2. Schematic of the particle impact tester.

Table 1. Experimental conditions.

\begin{tabular}{llll}
\hline Stage & $\begin{array}{l}\text { Nozzle diameter } \\
(\mathrm{mm})\end{array}$ & $\begin{array}{l}\text { Air flow velocity } \\
\text { below orifice }\left(\mathrm{m} \mathrm{s}^{-1}\right)\end{array}$ & $\begin{array}{l}\text { Cut off diameter of } \\
50 \% \text { collection }(\mu \mathrm{m})\end{array}$ \\
\hline $1^{\text {st }}$ & 5.3 & 15.4 & 11.4 \\
$2^{\text {nd }}$ & 3.5 & 35.4 & 6.11 \\
$3^{\text {rd }}$ & 2.7 & 59.5 & 4.14 \\
$4^{\text {th }}$ & 2.0 & 108.4 & 2.64 \\
$5^{\text {th }}$ & 1.6 & 169.4 & 1.89 \\
$6^{\text {th }}$ & 1.1 & 358.4 & 1.08 \\
\hline
\end{tabular}


considered to calculate the flow velocity in the nozzle and the theoretical cut off diameter of $50 \%$ collection (Okuda et al., 2018). This aspect was not considered because, unfortunately, the internal pressure of the cascade impactor could not be measured because of the physical restrictions of the apparatus. However, this was not considered a significant problem since the particles used were large enough compared with the theoretical cut off diameter of $50 \%$ collection.

\section{RESULTS AND DISCUSSION}

\subsection{XRD Pattern}

Fig. 3 shows the XRD pattern for different powder samples. The observed diffraction peaks were indexed to the unit cell of the terephthalic acid, and no difference in the peak positions among the samples was observed. Nevertheless, certain differences in the peak height among the samples were clearly observed. This result indicates a clear difference in the crystallinity among the samples. Table 2 summarizes the calculated crystallite size and the $50 \%$ particles diameter. Generally, the higher the crystallinity, the higher the energy required to break a crystal structure. Thus, it can be assumed that the particles' strength or, in other words, the particles' hardness satisfies the expression $A>B>C>D$.

\subsection{Single-particle Compression Test}

Fig. 4 shows a representative load-displacement curve of each sample for a single-particle compression test. The horizontal axis in the graph was normalized to a $50 \%$ particle diameter. It can be observed that the applied load increases at the early stage of particle compression. As the particle compression continues, the applied load does not vary with the displacement. At this point, it was considered that the particle fractured. Consequently, using this particular fracture load and Eq. (1), the fracture stress $S$ of a single particle was calculated. Generally, the fracture stress of a single particle exhibits a wide distribution. Similar behavior was observed in this experiment. Fig. 5 shows the relationship between the calculated crystallite size and a $50 \%$ fracture stress. In this

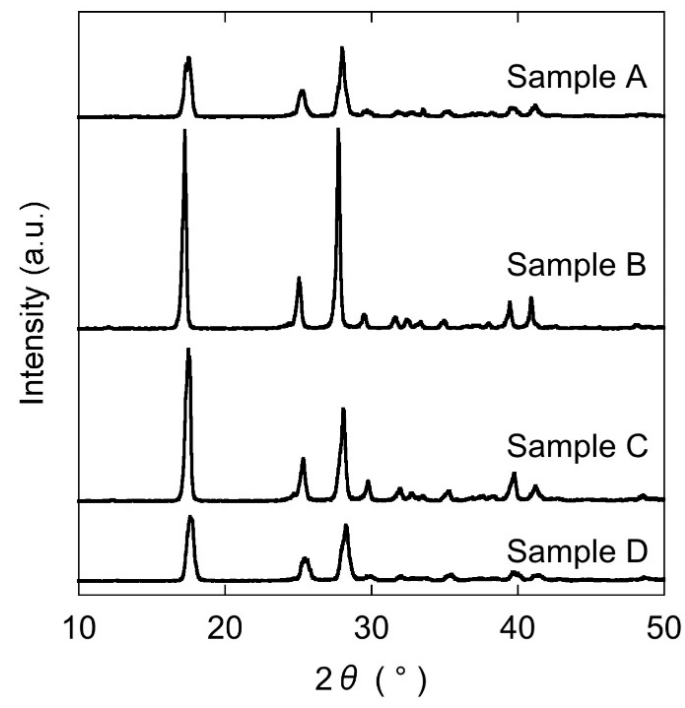

Fig. 3. XRD patterns for various terephthalic acid sample.

Table 2. Crystallite size and $50 \%$ particle diameter of various terephthalic acid powder.

\begin{tabular}{lll}
\hline Sample & Crystallite size $(\mathrm{nm})$ & $50 \%$ particle diameter $(\mu \mathrm{m})$ \\
\hline A & 406 & 150 \\
B & 290 & 130 \\
C & 210 & 152 \\
D & 150 & 181 \\
\hline
\end{tabular}




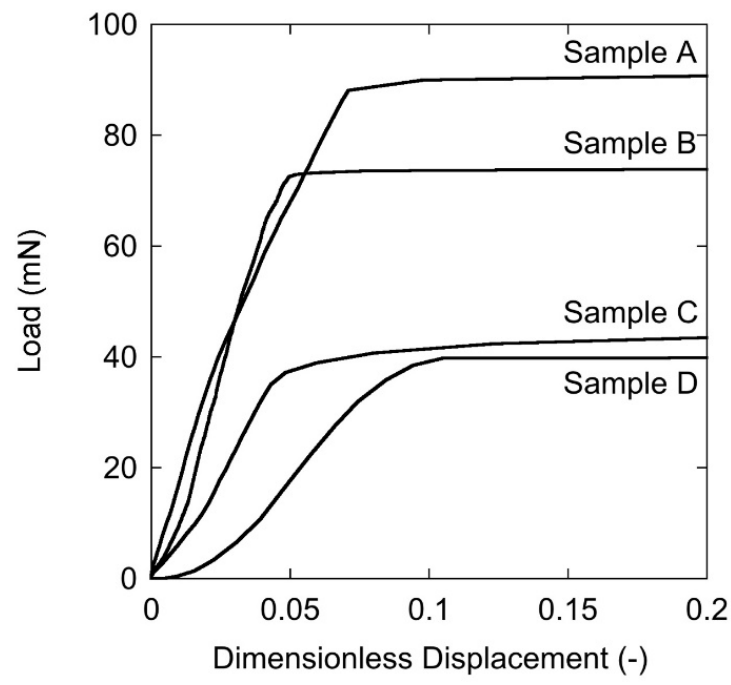

Fig. 4. Examples of load-displacement curves for various terephthalic acid sample.

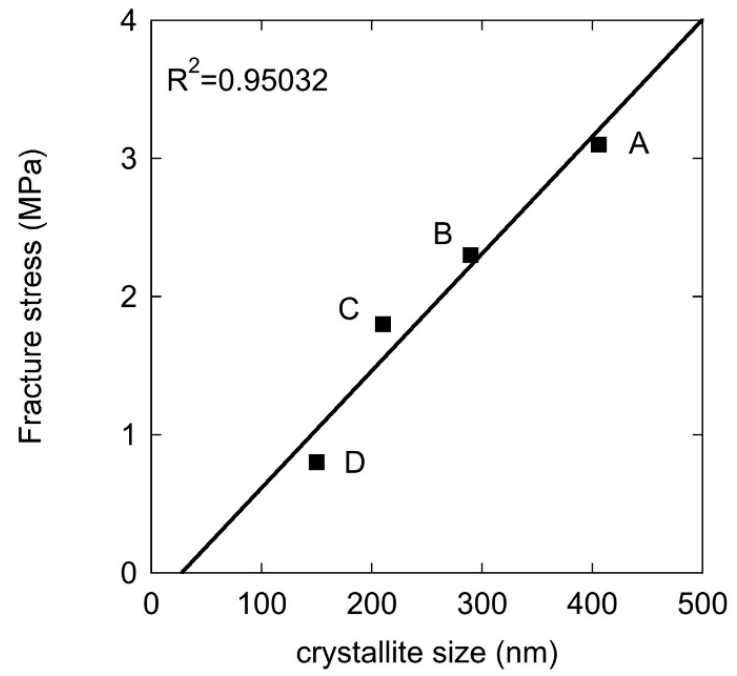

Fig. 5. Effect of crystallite size on fracture stress of single terephthalic acid particle.

figure, the straight line is a regression line obtained by applying the least-squares method. There is a correlation of the crystallite with the $50 \%$ fracture stress. Thus, the assumption that a particle's strength depends on the order of its crystallinity was confirmed.

\subsection{Particle Impact Test}

Fig. 6 shows the relationship between the calculated airflow velocities in the cascade impactor nozzle with the $50 \%$ particle diameter after performing the particle impact test. In this figure, the solid line trend can be easily explained. For each sample, its $50 \%$ particle diameter decreases at the flow velocity value indicated by a dashed line. It was assumed that many particles fractured at this point. The particles used in this experiment were relatively large; their Stokes number was over 450 at the maximum nozzle diameter condition. It can be considered that a particle's impact velocity is the same as the airflow velocity. Thus, this velocity was defined as the critical fracture velocity. Fig. 7(a) shows the relationship between the critical fracture velocity and the crystallite size of each particle sample. A correlation of the critical fracture velocity with the crystallite size can be observed. However, its $R^{2}$ value is lower than 0.95 . This can be due to the differences in the average particle size among the samples. To exclude the effect of particle size, the kinetic energy $E(J)$ of particles at their fracture point was calculated using the following equation, where 
(a)

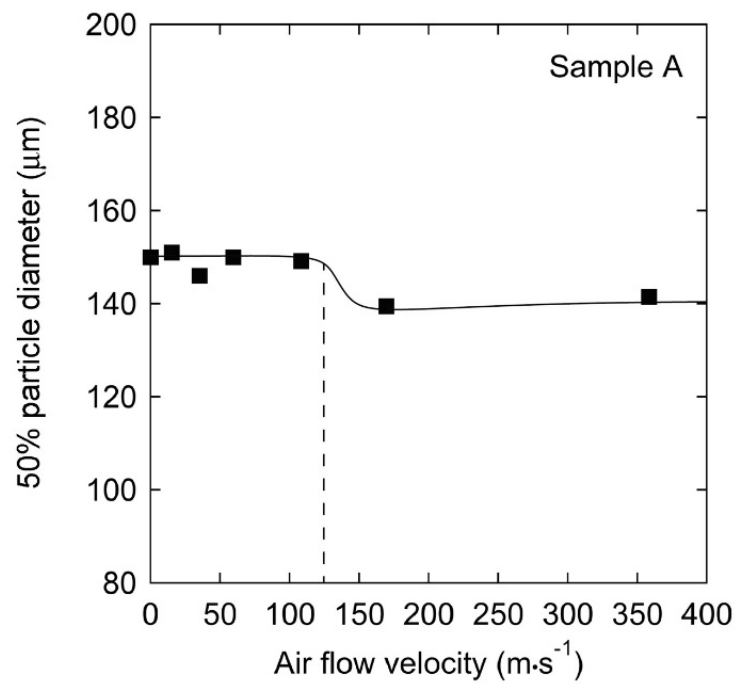

(c)

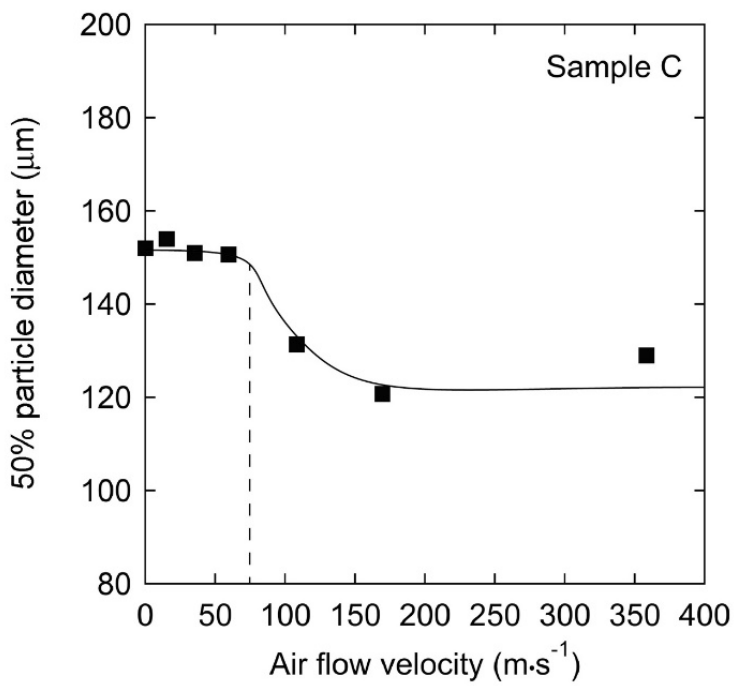

(b)

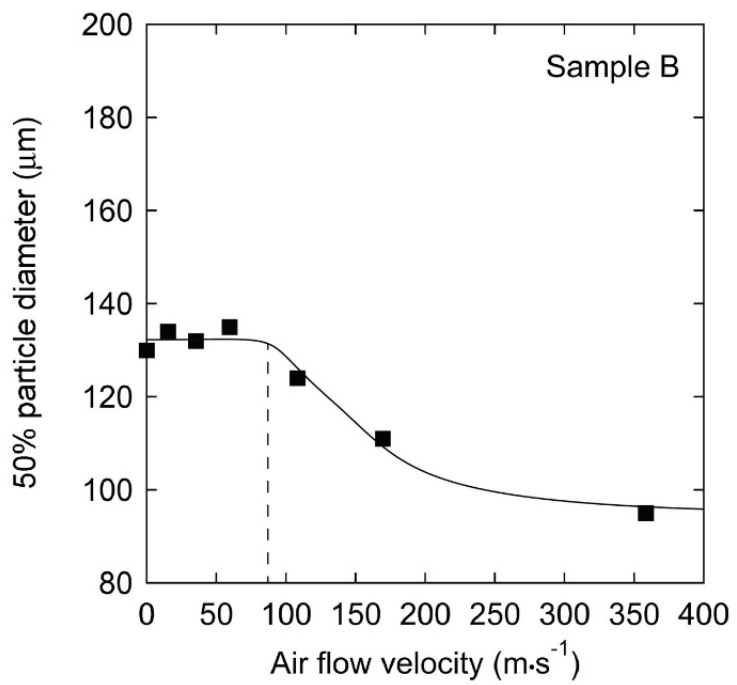

(d)

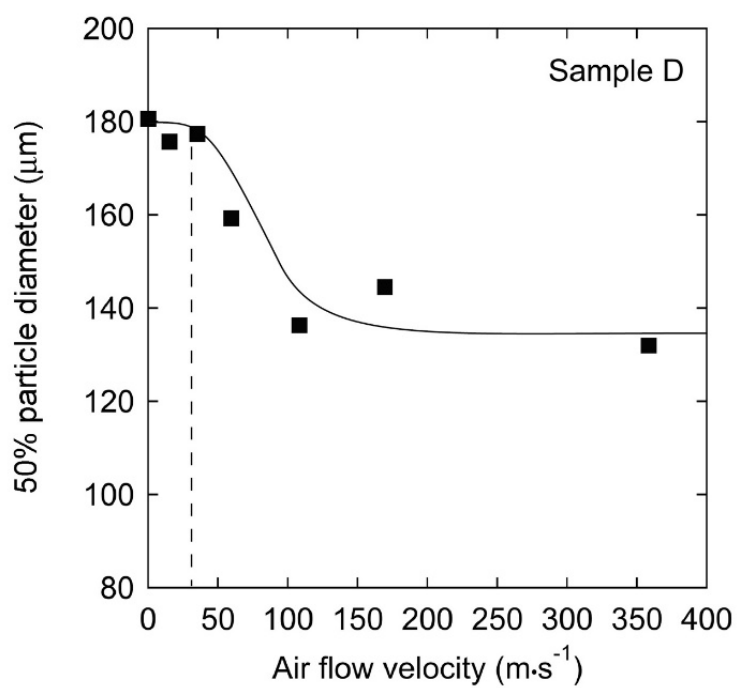

Fig. 6. Effect of air flow velocity on $50 \%$ particle diameter for various samples, (a) Sample A, (b) Sample B, (c) Sample C, (d) Sample D.

(a)

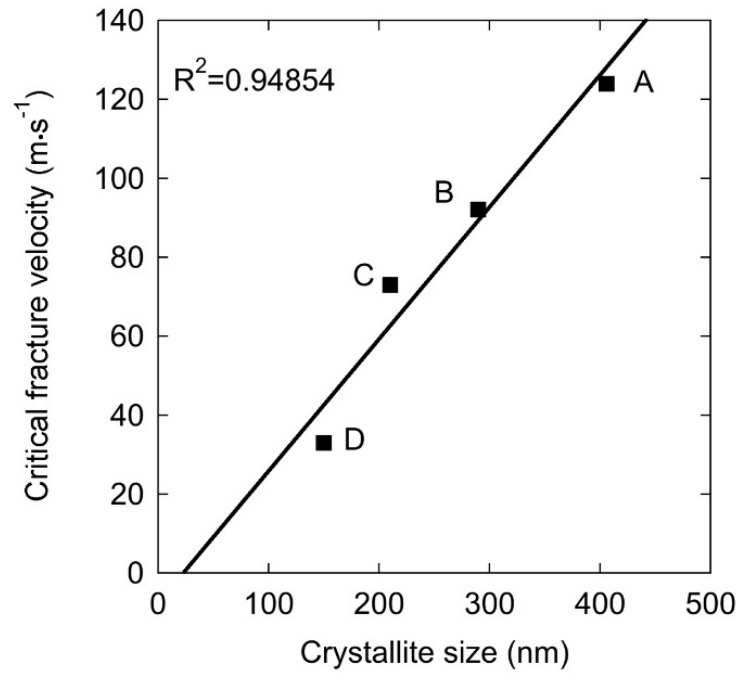

(b)

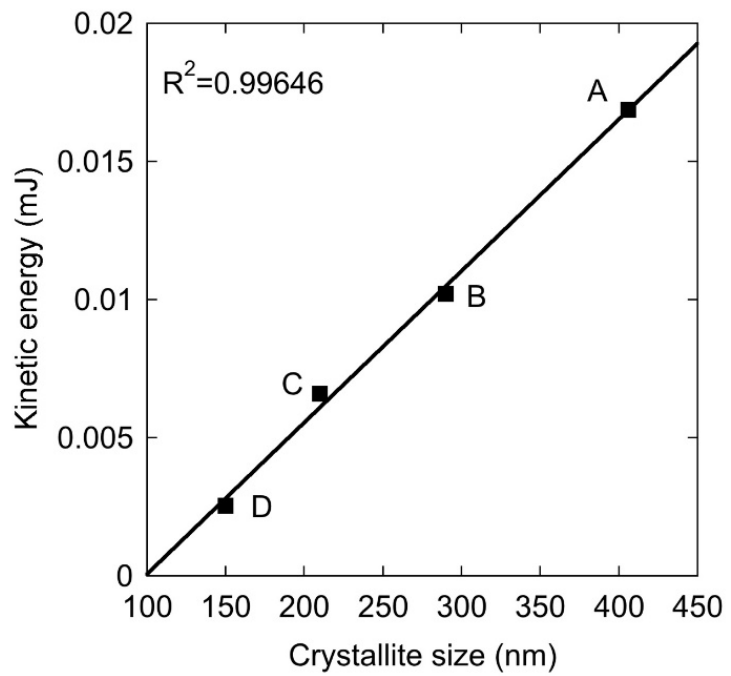

Fig. 7. Relation between (a) critical fracture velocity or (b) kinetic energy of single particle and crystallite size. 
$v_{\mathrm{c}}\left(\mathrm{m} \mathrm{s}^{-1}\right)$ is the critical fracture velocity, $x(\mathrm{~m})$ is the average particle diameter, and $\rho_{\mathrm{p}}\left(\mathrm{kg} \mathrm{m}^{-3}\right)$ is the particle density:

$E=\frac{1}{2} \cdot \frac{\pi}{6} x^{3} \rho_{p} \cdot v_{c}^{2}$

Fig. 7(b) shows the kinetic energy of a single particle at its fracture point as a function of the particle's crystallite size. The kinetic energy of a single particle exhibits a good correlation with the crystallite size, and its $R^{2}$ value is higher than 0.99 . Using this result, a particle's fracture velocity can be estimated from its crystallite size to prevent particle fracture.

Next, the effect of the impact angle on the critical fracture velocity was investigated. Particle B was selected as a sample because its fracture stress value was approximately the average among the fracture stress values of the four samples. Fig. 8 shows the relationship between the impact angle and the critical fracture velocity. The solid line in Fig. 8 exhibits a trend, which can be easily explained. It is observed that the particle fractured more easily at impact angles below $45^{\circ}$. For an impact angle in the $45^{\circ}-90^{\circ}$ range, the lower the impact angle, the lower the critical fracture velocity is. The single-particle fracturing under static and biaxial compression conditions was analyzed in Satone et al. (2017), where it was found that the required load for particle fracturing under a biaxial compression was less than that under a uniaxial compression. Under the biaxial compression with equal loads applied on the two axes, the angle of the resultant force formed by the two axes was $45^{\circ}$, and the required load for particle fracturing exhibited its lowest value. This result may be attributed to the following. The particle compressed along one axis deformed into a flat shape similar to that of a "Go stone" since the deformed material was able to move freely from compression along the two axes. The biaxially compressed granules deformed only in one free direction; however, their shape was spheroid, similar to that of a rugby ball. Under the biaxial compression, the granule material exhibited fewer degrees of freedom to disperse the compression load compared with the uniaxial compression case. Fracturing occurred even at low forces, as the biaxial load became unbalanced.

When a particle impacted the wall vertically, the particle was deformed in the two axes without moving direction. Its shape became flat, similar to that in the uniaxial compression. On the other hand, when a particle impacted the wall diagonally, the particle could be deformed only one axis. Its shape became spheroid, similar to that in the biaxial compression. This was considered as the reason that the particle fractured more easily at impact angles below $45^{\circ}$. Nevertheless, the deformation behavior of the impacted particle can only be speculated. We are in the process of investigating this topic using bigger particles and a high-speed video camera. When the impact angle is over $45^{\circ}$, it is considered that the particle could not collide with the wall; instead, it slid on the wall's surface. Thus, the particle could not easily fracture in this case

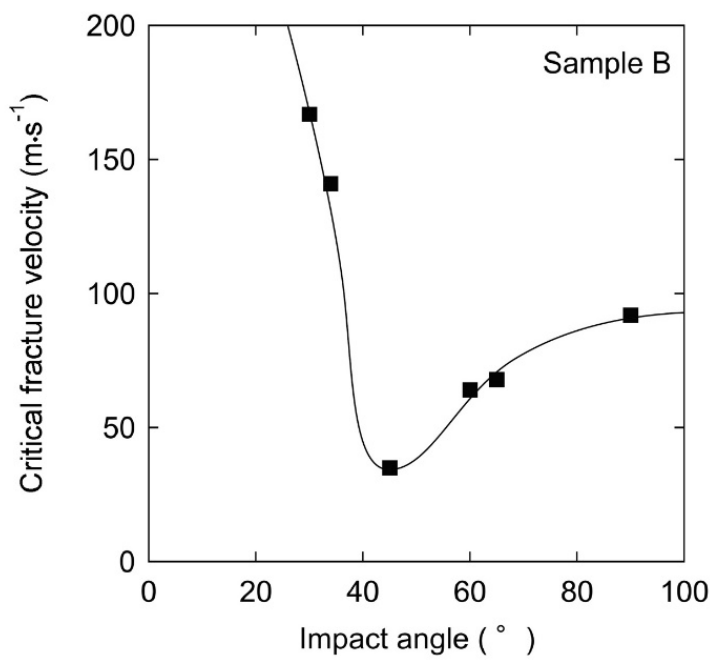

Fig. 8. Effect of impact angle of particle on critical fracture velocity. 
since the impact force could hardly be transmitted to the particle. In various real industrial processes involving particle movement, such as a powder pneumatic transportation system, there is an empirical law stating that particle fracturing occurs at the elbow of a pipe, where the airflow direction changes drastically. In this case, the particle will not always impact the inner wall vertically. The particle's trajectory changes because of various factors such as the elbow curvature radius, the elbow angle, and the insufficient Stokes number of the particle. Among these effects, the particle can impact the inner wall from various angles. This consideration is qualitatively related to the above empirical law.

\section{CONCLUSIONS}

In this study, the effect of terephthalic acid particle properties on particle fracture phenomena was explored. Furthermore, the fracturing characteristics in real processes were determined by investigating the effect of the particle impact angle on particle fracture phenomena. The results indicated that 1 ) the crystallite size is related to the fracture stress of a particle, 2) the crystallite size is related to the critical fracture velocity and the kinetic energy of the particle, and 3) a particle fractures more easily at impact angles below $45^{\circ}$. Using these results, the occurrence of particle fracturing in a powder pneumatic transportation system can be predicted by measuring the crystallite size of a particle and calculating the flow field.

\section{ACKNOWLEDGMENTS}

This work was supported by JSPS KAKENHI Grant Numbers JP19H04313, JP20K05200, the Japan Science and Technology Agency's Adaptable and Seamless Technology Transfer Program, Target-driven R\&D (A-STEP) Stage I, Industry Needs Response Type.

\section{REFERENCES}

Bolton, M.D., Nakata, Y., Cheng, Y.P. (2008). Micro- and macro-mechanical behaviour of DEM crushable materials. Géotechnique 58, 471-480. https://doi.org/10.1680/geot.2008.58.6.471

Chau, K.T., Wei, X.X., Wong, R.H.C., Yu, T.X. (2000). Fragmentation of brittle spheres under static and dynamic compressions: Experiments and analyses. Mech. Mater. 32, 543-554. https://doi.org/10.1016/S0167-6636(00)00026-0

Chaudhri, M.M. (2004). Impact breakage of semi-brittle spheres. Powder Technol. 143, 31-40. https://doi.org/10.1016/j.powtec.2004.04.006

Golchert, D., Moreno, R., Ghadiri, M., Litster, J. (2004). Effect of granule morphology on breakage behaviour during compression. Powder Technol. 143, 84-96. https://doi.org/10.1016/j. powtec.2004.04.032

Griffith, A.A. (1924). The theory of rupture, in: Biereno, C.B., Burgers, J.M. (Eds.), Proceedings of the first International Congress for Applied Mechanics, Delft, pp. 55-63.

Gundepudi, M.K., Sankar, B.V., Mecholsky Jr., J.J., Clupper, D.C. (1997). Stress analysis of brittle spheres under multiaxial loading Powder Technol. 94, 153-161. https://doi.org/10.1016/ s0032-5910(97)03307-x

Hiramatsu, Y., Oka, Y., Kiyama, H. (1965). Rapid determination of tensile strength of rocks with irregular test pieces. Nihon Kogyokai-shi 81, 1024-1030. https://doi.org/10.2473/shigentosoz ai1953.81.932_1024

JIS R1639-5 (2007). Test methods of properties of fine ceramic granules Part 5: Compressive strength of a single granule, Japanese Industrial Standards Committee. https://www.jisc.go.jp/ eng/index.html

Kanda, Y. (1996). Investigation on history of research and technique in comminution. J. Soc. Mater. Eng. Resour. Jpn. 9, 87-110. https://doi.org/10.5188/jsmerj.9.2_87

Khanal, M., Schubert, W., Tomas, J. (2005). Experiment and simulation of breakage of particle compounds under compressive loading. Part. Sci. Technol. 23, 387-394. https://doi.org/ $10.1080 / 02726350500213010$ 
Okuda, T., Ehara, K., lida, K. (2018). 6. Classification-II: Classification using inertial and centrifugal forces. Earozoru Kenkyu 32, 276-288. https://doi.org/10.11203/jar.32.276

Pitchumani, R., Zhupanska, O., Meesters, G.M.H., Scarlett, B. (2004). Measurement and characterization of particle strength using a new robotic compression tester. Powder Technol. 143, 56-64. https://doi.org/10.1016/j.powtec.2004.04.007

Satone, H., limura, K., Teraoka, T., Hanafusa, T., Hisatani, S., Nishiwaki, M., Suzuki, M. (2017). Analysis of granule fracture under biaxial compression. Ceram. Int. 43, 16835-16842. https://doi.org/10.1016/j.ceramint.2017.09.081

Samimi, A., Hassanpour, A., Ghadiri, M. (2005). Single and bulk compression of soft granules: Experimental and DEM evaluation. Chem. Eng. Sci. 60, 3993-4004. https://doi.org/ 10.1016/j.ces.2005.02.036

Tsoungui, O., Vallet, D., Charmet, J.C. (1999). Numerical model of crushing of grains inside twodimensional granular materials. Powder Technol. 105, 190-198. https://doi.org/10.1016/ S0032-5910(99)00137-0

Yashima, S., Saito, F. (1979). Size effects of particle compressive strength on single particle crushing. J. Soc. Powder Technol. Jpn. 16, 714-720. https://doi.org/10.4164/sptj.16.714 